\title{
Formulation and Experimental Verification of an Axisymmetric Finite-Element Structural Analysis
}

\author{
R. A. Mitchell, R. M. Woolley, and C. R. Fisher* \\ Institute for Basic Standards, National Bureau of Standards, Washington, D.C. 20234
}

(September 29, 1971)

\begin{abstract}
A finite-element structural analysis is described for application to problems in which geometry and loading are axisymmetric and material properties are isotropic elastic. An attempt to minimize restrictions imposed on the shape and orientation of the triangular finite-elements has been largely successful. This facilitates use of the analysis, with automatic finite-element mesh generation, in parameter or optimization studies. A series of laboratory tests to verify the analysis are described in which the magnitude and distribution of boundary loading was known within narrow limits.
\end{abstract}

Key words: Axisymmetric; elastic; experiment; finite-element analysis; force transducer; stiffness matrix; structural analysis.

\section{Introduction}

In the structural optimization of a force transducer of the elastic column type, one is concerned with relationships between surface strains and such parameters as boundary loading, dimensions, and material properties. Finite-element analysis is probably the most versatile structural analysis method currently available for studying these relationships. The finite-element formulation described here was developed for application to such optimization problems in which both the geometry and loading are axisymmetric and the materials are isotropic elastic. This class of problems is analytically two-dimensional. Ring-shaped finite elements of triangular cross section and a global Cartesian coordinate system are used in the analysis. This approach generally follows the outlines given by Clough [1, 2], ${ }^{1}$ Rashid [2, 3, 4], and Wilson [5].

For efficient structural optimization it is necessary to program the computer to generate automatically the mesh that subdivides a structure into a network of finite elements. To facilitate this, an attempt has been made in this formulation to keep to a minimum the restrictions imposed on shape and orientation of the triangular elements. Explicit integration, used here to develop general formulas for the stiffness of finite elements, yields logarithmic terms which require special treatment to avoid excessive errors when one side of a triangular element is oriented at

\footnotetext{
${ }^{*}$ Present address: Naval Scientific and Technical Intelligence Center, Washington, D.C. 20390

${ }^{1}$ Figures in brackets indicate literature references at end of paper.
}

a small non-zero angle with respect to the axis of symmetry. The special treatment adopted here is to expand the logarithmic integrals into rapidly converging infinite series. Although the stiffness integrals are rather intricate in both the logarithmic and the series forms, they do avoid the need for numerical integration.

An experimental verification of the analysis was obtained by a series of tests conducted on the structural body of a force transducer (load cell) made of 18 percent nickel 250 grade maraging steel. Axial compressive load was applied to the load-cell body. The load was distributed over a small circular area at the top and over a narrow ring-shaped area at the bottom. Thus, the location of applied load was known within narrow limits. Strains on the surface of the load-cell body, known to vary significantly with load location or distribution, were measured and found to be in good agreement with the finite-element analysis.

\section{Finite-Element Formulation}

In a finite-element analysis the continuum structure is subdivided into a network of elements that are connected to adjacent elements only at common nodal points. Elastic displacements within the individual elements are assumed to be defined by generalized functions that assure displacement compatibility along common boundaries of adjacent elements. The stiffness matrix of each element, relating nodal point forces and displacements, is then computed in terms of the assumed displacement functions, dimensions, and material properties of the element. The stiffness matrix for the entire structure, relating 


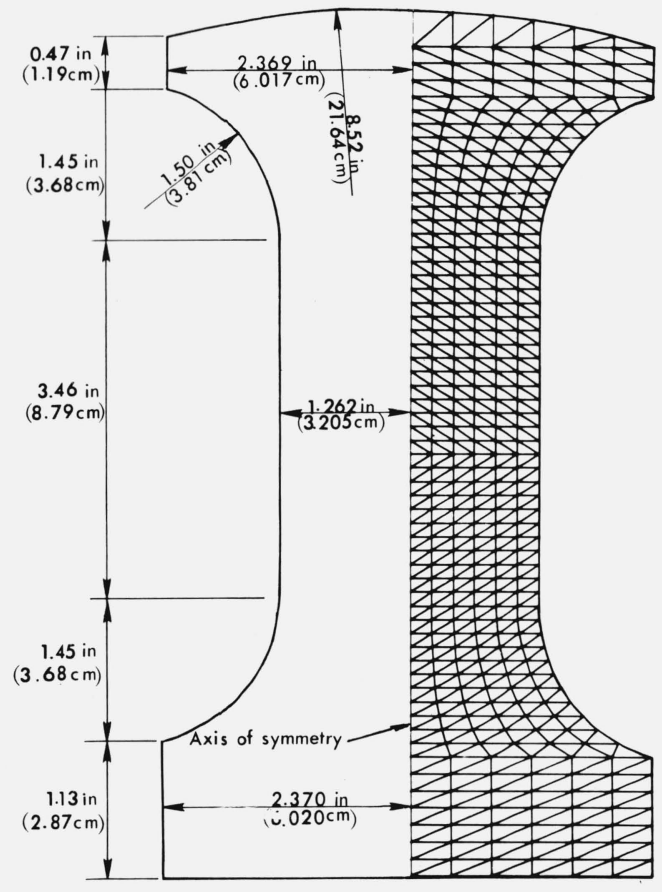

FigURE 1. Longitudinal cross section of load-cell body used in laboratory tests

applied external forces to nodal point displacements throughout the structure, is formed by superposing the element stiffness matrices.

A longitudinal cross section of the load-cell body used in the laboratory tests is shown in figure 1 . The figure illustrates a triangular finite element mesh. A similar but finer mesh was used for the analyses that were correlated with the laboratory tests.

The triangular cross section of a general element is shown in figure 2. Each nodal point (actually, a nodal circle in the axisymmetric case) has independent displacement components in the $r$ and $z$ directions. The generalized displacement functions assumed for this formulation are given by

$$
\left\{\begin{array}{l}
u \\
v
\end{array}\right\}=\left[\begin{array}{ccccc}
1 & r-r_{i} z-z_{i} & 0 & 0 & 0 \\
0 & 0 & 0 & 1 & r-r_{i} z-z_{i}
\end{array}\right]\left\{\begin{array}{c}
\alpha_{1} \\
\alpha_{2} \\
\alpha_{3} \\
\alpha_{4} \\
\alpha_{5} \\
\alpha_{6}
\end{array}\right\}
$$

in which

$u=$ displacement in the $r$ direction of any point in the element,

$v=$ displacement in the $z$ direction of any point in and

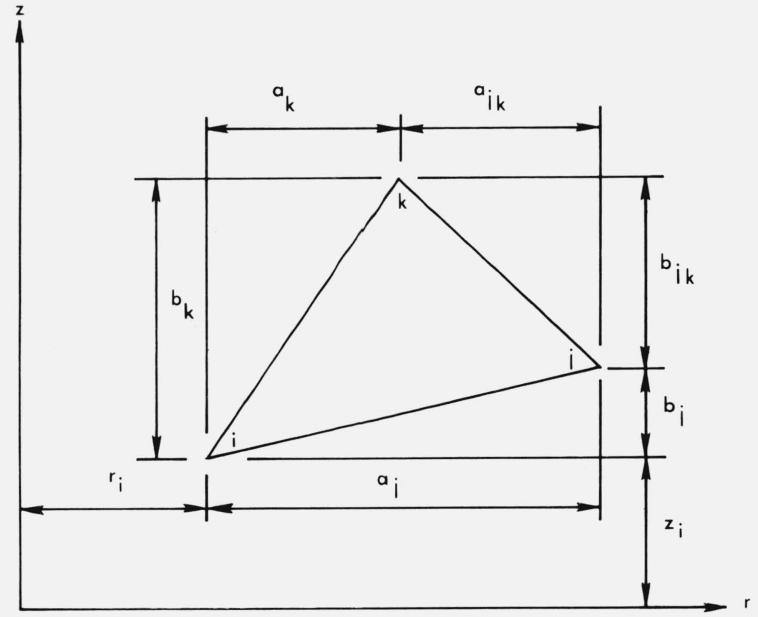

FIGURE 2. Cross section of a general axisymmetric finite element.

$\alpha_{n}=$ a generalized coordinate

A generalized coordinate, $\alpha_{n}$, represents either the $r$ or $z$ component of displacement of nodal point $i$ or a rate of change of a displacement component with respect to $r$ or $z$. The assumed displacement functions give linear variations in displacement along element boundaries and, therefore, complete displacement compatibility between adjacent elements. Stresses within adjacent elements are not, in general, in equilibrium along common boundaries, but the resultant forces acting at nodal points are required to be in equilibrium. Substitution of nodal point coordinates (defined in fig. 2) into eq (1) gives the nodal point displacements in terms of the generalized coordinates.

$$
\left.\left\{\begin{array}{l}
u_{i} \\
u_{j} \\
u_{k} \\
v_{i} \\
v_{j} \\
v_{k}
\end{array}\right\}=\left[\begin{array}{llllll}
1 & 0 & 0 & 0 & 0 & 0 \\
1 & a_{j} & b_{j} & 0 & 0 & 0 \\
1 & a_{k} & b_{k} & 0 & 0 & 0 \\
0 & 0 & 0 & 1 & 0 & 0 \\
0 & 0 & 0 & 1 & a_{j} & b_{j} \\
0 & 0 & 0 & 1 & a_{k} & b_{k}
\end{array}\right] \quad \begin{array}{c}
\alpha_{1} \\
\alpha_{2} \\
\alpha_{3} \\
\alpha_{4} \\
\alpha_{5} \\
\alpha_{6}
\end{array}\right\},
$$

or

$$
\{w\}=[A]\{\alpha\} \cdot
$$

Element strains are obtained by applying the definitions of strain from elasticity theory [6] to eq (1) as follows:

$$
\left\{\begin{array}{l}
\epsilon_{r} \\
\epsilon_{\theta} \\
\epsilon_{z} \\
\gamma_{r z}
\end{array}\right\}=\left\{\begin{array}{l}
\frac{\partial u}{\partial r} \\
\frac{u}{r} \\
\frac{\partial v}{\partial z} \\
\frac{\partial u}{\partial z}+\frac{\partial v}{\partial r}
\end{array}\right\}=\left[\begin{array}{cccccc}
0 & 1 & 0 & 0 & 0 & 0 \\
\frac{1}{r} & \frac{r-r_{i}}{r} & \frac{z-z_{i}}{r} & 0 & 0 & 0 \\
0 & 0 & 0 & 0 & 0 & 1 \\
0 & 0 & 1 & 0 & 1 & 0
\end{array}\right]\left\{\begin{array}{c}
\alpha_{1} \\
\alpha_{2} \\
\alpha_{3} \\
\alpha_{4} \\
\alpha_{5} \\
\alpha_{6}
\end{array}\right\}
$$


The assumed stress-strain relationship for isotropic elastic materials is

$$
\left.\left\{\begin{array}{c}
\sigma_{r} \\
\sigma_{\theta} \\
\sigma_{z} \\
\tau_{r z}
\end{array}\right\}=\frac{\nu E}{(1+\nu)(1-2 \nu)}\left[\begin{array}{cccc}
\frac{1-\nu}{\nu} & 1 & 1 & 0 \\
1 & \frac{1-\nu}{\nu} & 1 & 0 \\
1 & 1 & \frac{1-\nu}{\nu} & 0 \\
0 & 0 & 0 & \frac{1-2 \nu}{2 \nu}
\end{array}\right] \mid \begin{array}{c}
\epsilon_{r} \\
\epsilon_{\theta} \\
\epsilon_{z} \\
\gamma_{r z}
\end{array}\right\},(6)
$$

or $\quad\{\sigma\}=[D]\{\epsilon\}$,

in which $E=$ Modulus of elasticity

and $\quad \nu=$ Poisson's ratio.

By a process of equating internal to external virtual work, using eqs (3), (5), and (7), the general equation for nodal point stiffness of an element can be shown to be (see ref $1,2,3$ and 7 )

$$
[k]=\left[A^{-1}\right]^{T}\left(\int_{\text {Volume }}[B]^{T}[D][B] d V\right)\left[A^{-1}\right],(8)
$$

or

$$
[k]=\left[A^{-1}\right]^{T}[\bar{k}]\left[A^{-1}\right] .
$$

In eq (8), $d V$ is a differential volume within the element. Integration is performed over the volume of the element, and the superscripts $T$ and -1 indicate matrix transposition and inversion, respectively. The stiffness matrix $[k]$ relates the six components of force $\{f\}$ acting at the three nodal points of a triangular element to the six components of nodal point displacement $\{w\}$ by

$$
\{f\}=[k]\{w\} .
$$

Using $r, z-z_{i}$, and $\theta$ as the variables of integration, and defining the integrals of the functions of these variables by

$I\left(f\left(r, z-z_{i}, \theta\right)\right) \equiv 2 \pi \int_{r} \int_{z-z_{i}} f\left(r, z-z_{i}\right) r d\left(z-z_{i}\right) d r,(11)$

the nonzero elements of $[\bar{k}]$, eq (9), are

$$
\bar{k}_{11}=\frac{(1-\nu) E}{(1+\nu)(1-2 \nu)} I\left(\frac{1}{r^{2}}\right)
$$

$\bar{k}_{12}=\bar{k}_{21}=\frac{E}{(1+\nu)(1-2 \nu)} I\left(\frac{1}{r}\right)$

$$
-\frac{(1-\nu) E r_{i}}{(1+\nu)(1-2 \nu)} I\left(\frac{1}{r^{2}}\right) \quad I_{2}\left(f\left(r, z-z_{i}\right)\right)=2 \pi \int_{r_{k}}^{r_{j}} \int_{z_{i j}}^{z_{j k}} f\left(r, z-z_{i}\right) r d\left(z-z_{i}\right) d r
$$




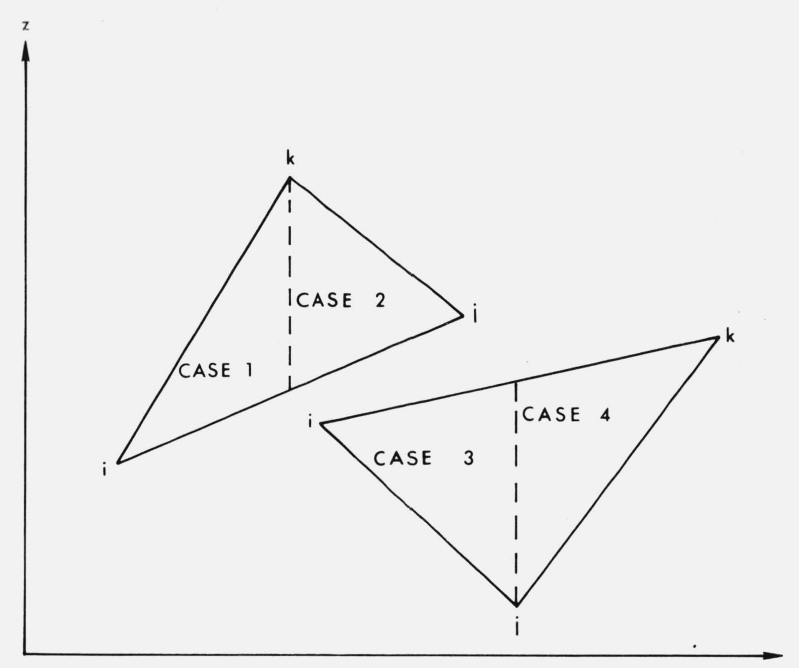

Figure 3. Four different integration limit cases.

Case 3:

$I_{3}\left(f\left(r, z-z_{i}\right)\right)=2 \pi \int_{r_{i}}^{r_{j}} \int_{z_{i j}}^{z_{i k}} f\left(r, z-z_{i}\right) r d\left(z-z_{i}\right) d r$

Case 4:

$I_{4}\left(f\left(r, z-z_{i}\right)\right)=2 \pi \int_{r_{j}}^{r_{k}} \int_{z_{j k}}^{z_{i k}} f\left(r, z-z_{i}\right) r d\left(z-z_{i}\right) d r$

in which the limit notation $z_{i j}$ represents the value of $z-z_{i}$ along line $i-j$. Three of the functions in $[\bar{k}]$ integrate directly, for either orientation of side $j-k$, to

$$
\begin{gathered}
I(1)=\frac{2}{3} \pi A\left(r_{i}+r_{j}+r_{k}\right), \\
I\left(\frac{1}{r}\right)=2 \pi A,
\end{gathered}
$$

and

$$
I\left(\frac{z-z_{i}}{r}\right)=\frac{2}{3} \pi A\left(b_{j}+b_{k}\right)
$$

in which $A=\frac{1}{2}\left(a_{j} b_{k}-a_{k} b_{j}\right)=$ area of the element cross section.
The integrals of the other three functions in $[\bar{k}]$, for the four cases shown in figure 3 , are

$$
\begin{gathered}
I_{1}\left(\frac{1}{r^{2}}\right)=\frac{4 \pi A}{a_{j}}\left(1-\frac{r_{i}}{a_{k}} \ln \frac{r_{k}}{r_{i}}\right) \\
I_{2}\left(\frac{1}{r^{2}}\right)=-\frac{4 \pi A}{a_{j}}\left(1-\frac{r_{j}}{a_{j k}} \ln \frac{r_{j}}{r_{k}}\right) \\
I_{3}\left(\frac{1}{r^{2}}\right)=\frac{4 \pi A}{a_{k}}\left(1-\frac{r_{i}}{a_{j}} \ln \frac{r_{j}}{r_{i}}\right) \\
I_{4}\left(\frac{1}{r^{2}}\right)=-\frac{4 \pi A}{a_{k}}\left(1-\frac{r_{k}}{a_{j k}} \ln \frac{r_{j}}{r_{k}}\right) \\
I_{1}\left(\frac{z-z_{i}}{r^{2}}\right)=2 \pi A\left(\frac{a_{j} b_{k}+a_{k} b_{j}}{a_{j}^{2}}\right)\left[\frac{1}{2}-\frac{r_{i}}{a_{k}}\left(1-\frac{r_{i}}{a_{k}} \ln \frac{r_{k}}{r_{i}}\right)\right]
\end{gathered}
$$

$I_{2}\left(\frac{z-z_{i}}{r^{2}}\right)$

$$
\begin{aligned}
& =\frac{2 \pi A}{a_{j k}^{2}}\left[2 A-2 b_{j k} r_{i}+r_{i}^{2}\left(\frac{a_{j} b_{k}+a_{k} b_{j}-2 a_{j} b_{j}}{a_{j}^{2}}\right)\right] \ln \frac{r_{j}}{r_{k}} \\
& +\frac{\pi A}{a_{j}^{2}}\left[\frac{2 a_{j}^{2} b_{j k}}{a_{j k}}-a_{j} b_{k}-a_{k} b_{j}-2 r_{i}\left(\frac{a_{j} b_{k}+a_{k} b_{j}-2 a_{j} b_{j}}{a_{j k}}\right)\right]
\end{aligned}
$$

$$
I_{3}\left(\frac{z-z_{i}}{r^{2}}\right)=2 \pi A\left(\frac{a_{j} b_{k}+a_{k} b_{j}}{a_{k}^{2}}\right)\left[\frac{1}{2}-\frac{r_{i}}{a_{j}}\left(1-\frac{r_{i}}{a_{j}} \ln \frac{r_{j}}{r_{i}}\right)\right]
$$

$I_{4}\left(\frac{z-z_{i}}{r^{2}}\right)$

$$
\begin{aligned}
& =\frac{2 \pi A}{a_{j k}^{2}}\left[2 A-2 b_{j k} r_{i}-r_{i}^{2}\left(\frac{a_{j} b_{k}+a_{k} b_{j}-2 a_{k} b_{k}}{a_{k}^{2}}\right)\right] \ln \frac{r_{j}}{r_{k}} \\
& +\frac{\pi A}{a_{k}^{2}}\left[\frac{2 a_{k}^{2} b_{j k}}{a_{j k}}-a_{j} b_{k}-a_{k} b_{j}+2 r_{i}\left(\frac{a_{j} b_{k}+a_{k} b_{j}-2 a_{k} b_{k}}{a_{j k}}\right)\right]
\end{aligned}
$$$$
I_{1}\left(\frac{\left(z-z_{i}\right)^{2}}{r^{2}}\right)=4 \pi A\left(\frac{a_{j}^{2} b_{k}^{2}+a_{k}^{2} b_{j}^{2}+a_{j} a_{k} b_{j} b_{k}}{a_{j}^{3}}\right)\left[\frac{1}{9}-\frac{r_{i}}{6 a_{k}}\right.
$$

$$
\left.+\frac{r_{i}^{2}}{3 a_{k}^{2}}\left(1-\frac{r_{i}}{a_{k}} \ln \frac{r_{k}}{r_{i}}\right)\right]
$$

$$
\begin{aligned}
I_{2}\left(\frac{\left(z-z_{i}\right)^{2}}{r^{2}}\right)= & \frac{4 \pi A}{3 a_{i k}^{3}}\left[4 A^{2}-6 A b_{j k} r_{i}+3 b_{j k}^{2} r_{i}^{2}+r_{i}^{3}\left(\frac{4 A^{2}+3 a_{j} a_{j k} b_{j} b_{j k}}{a_{i}^{3}}\right)\right] \ln \frac{r_{j}}{r_{k}} \\
& +\frac{2 \pi A}{a_{j k}^{2}}\left[b_{j k}\left(2 A+a_{j} b_{j}-a_{k} b_{k}\right)-2 b_{j k}^{2} r_{i}+r_{i}\left(a_{j}+a_{k}\right)\left(\frac{4 A^{2}+3 a_{j} a_{j k} b_{j} b_{j k}}{a_{j}^{3}}\right)\right] \\
& -\frac{4 \pi A}{9}\left(1+3 \frac{r_{j} r_{k}}{a_{j k}^{2}}\right)\left(\frac{4 A^{2}+3 a_{j} a_{j k} b_{j} b_{j k}}{a_{j}^{3}}\right)
\end{aligned}
$$




$$
\begin{aligned}
I_{3}\left(\frac{\left(z-z_{i}\right)^{2}}{r^{2}}\right)= & 4 \pi A\left(\frac{a_{j}^{2} b_{k}^{2}+a_{k}^{2} b_{j}^{2}+a_{j} a_{k} b_{j} b_{k}}{a_{k}^{3}}\right)\left[\frac{1}{9}-\frac{r_{i}}{6 a_{j}}+\frac{r_{i}^{2}}{3 a_{j}^{2}}\left(1-\frac{r_{i}}{a_{j}} \ln \frac{r_{j}}{r_{i}}\right)\right] \\
I_{4}\left(\frac{\left(z-z_{i}\right)^{2}}{r^{2}}\right)= & \frac{4 \pi A}{3 a_{j k}^{3}}\left[4 A^{2}-6 A b_{j k} r_{i}+3 b_{j k}^{2} r_{i}^{2}+r_{i}^{3}\left(\frac{4 A^{2}+3 a_{k} a_{j k} b_{k} b_{j k}}{a_{k}^{3}}\right)\right] \ln \frac{r_{j}}{r_{k}} \\
& +\frac{2 \pi A}{a_{j k}^{2}}\left[b_{j k}\left(2 A+a_{j} b_{j}-a_{k} b_{k}\right)-2 b_{j k}^{2} r_{i}+r_{i}\left(a_{j}+a_{k}\right)\left(\frac{4 A^{2}+3 a_{k} a_{j k} b_{k} b_{j k}}{a_{k}^{3}}\right)\right] \\
& -\frac{4 \pi A}{9}\left(1+3 \frac{r_{j} r_{k}}{a_{j k}^{2}}\right)\left(\frac{4 A^{2}+3 a_{k} a_{j k} b_{k} b_{j k}}{a_{k}^{3}}\right) .
\end{aligned}
$$

The logarithmic terms in the above integrals require special treatment to avoid excessive errors in numerical computations. By a limiting process employing L'Hospital's rule it can be shown that, as $r_{i}$ or both $r_{i}$ and $r_{k}$ tend to zero, the limit of the logarithmic term is zero. Therefore, if one or two nodal points of an element lie on the $z$ axis, the logarithmic terms are omitted from the computation. A more difficult computational problem is presented when one side of a finite element is oriented at a small nonzero angle relative to the $z$ axis. To permit the use of elements with a side so oriented, the logarithmic terms can be expanded in the series (Peirce's formula $768[8]$ )

$\ln (1+\chi)=\chi-\frac{\chi^{2}}{2}+\frac{\chi^{3}}{3}-\frac{\chi^{4}}{4}+\ldots+(-1)^{n-1} \frac{\chi^{n}}{n}+\ldots$

$$
\begin{aligned}
& I_{1}^{\prime}\left(\frac{1}{r^{2}}\right)=\frac{4 \pi A}{a_{j}} \sum_{n} \frac{(-1)^{n+1}}{n+1}\left(\frac{a_{k}}{r_{i}}\right)^{n} \\
& I_{2}^{\prime}\left(\frac{1}{r^{2}}\right)=-\frac{4 \pi A}{a_{j}} \sum_{n} \frac{1}{n+1}\left(\frac{a_{j k}}{r_{j}}\right)^{n} \\
& I_{3}^{\prime}\left(\frac{1}{r^{2}}\right)=\frac{4 \pi A}{a_{k}} \sum_{n} \frac{(-1)^{n+1}}{n+1}\left(\frac{a_{j}}{r_{i}}\right)^{n} \\
& I_{4}^{\prime}\left(\frac{1}{r^{2}}\right)=-\frac{4 \pi A}{a_{k}} \sum_{n} \frac{(-1)^{n+1}}{n+1}\left(\frac{a_{j k}}{r_{k}}\right)^{n}
\end{aligned}
$$
which converges in the interval $-1<\chi<1$. This $I_{1}^{\prime}\left(\frac{z-z_{i}}{r^{2}}\right)=2 \pi A\left(\frac{a_{j} b_{k}+a_{k} b_{j}}{a_{j}^{2}}\right) \sum_{n} \frac{(-1)^{n+1}}{n+2}\left(\frac{a_{k}}{r_{i}}\right)^{n}$
gives the following alternate forms of the integrals:

$$
\begin{aligned}
& I_{2}^{\prime}\left(\frac{z-z_{i}}{r^{2}}\right)=\frac{2 \pi A}{a_{j}^{2}}\left[2 A \sum_{n} \frac{1}{n+2}\left(\frac{a_{j k}}{r_{j}}\right)^{n}-2 b_{j} r_{i} \sum_{n} \frac{1}{n+1}\left(\frac{a_{j k}}{r_{j}}\right)^{n}+a_{j k} b_{j}\right] \\
& I_{3}^{\prime}\left(\frac{z-z_{i}}{r^{2}}\right)=2 \pi A\left(\frac{a_{j} b_{k}+a_{k} b_{j}}{a_{k}^{2}}\right) \sum_{n} \frac{(-1)^{n+1}}{n+2}\left(\frac{a_{j}}{r_{i}}\right)^{n} \\
& I_{4}^{\prime}\left(\frac{z-z_{i}}{r^{2}}\right)=\frac{2 \pi A}{a_{k}^{2}}\left[2 A \sum_{n} \frac{(-1)^{n+1}}{n+2}\left(\frac{a_{j k}}{r_{k}}\right)^{n}+2 b_{k} r_{i} \sum_{n} \frac{(-1)^{n+1}}{n+1}\left(\frac{a_{j k}}{r_{k}}\right)^{n}-b_{k} a_{j k}\right] \\
& I_{1}^{\prime}\left(\frac{\left(z-z_{i}\right)^{2}}{r^{2}}\right)=4 \pi A\left(\frac{a_{j}^{2} b_{k}^{2}+a_{k}^{2} b_{j}^{2}+a_{j} a_{k} b_{j} b_{k}}{a_{j}^{3}}\right) \sum_{n} \frac{(-1)^{n+1}}{n+3}\left(\frac{a_{k}}{r_{i}}\right)^{n}
\end{aligned}
$$

If $b_{j}=0$,

$I_{2}^{\prime}\left(\frac{\left(z-z_{i}\right)^{2}}{r^{2}}\right)=\frac{4 \pi A a_{j}^{2} b_{k}^{2}}{3 a_{j}^{3}} \sum_{n} \frac{1}{n+3}\left(\frac{a_{j k}}{r_{j}}\right)^{n}$

If $b_{k}=0$,

$$
\begin{aligned}
& I_{2}^{\prime}\left(\frac{\left(z-z_{i}\right)^{2}}{r^{2}}\right)=\frac{4 \pi A b_{j}^{2}}{3 a_{j}^{3}}\left[a_{k}^{2} \sum_{n} \frac{1}{n+3}\left(\frac{a_{j k}}{r_{j}}\right)^{n}-3 a_{j} r_{i} \sum_{n}\left(\frac{1}{n+1}-\frac{1}{n+2}\right)\left(\frac{a_{j k}}{r_{j}}\right)^{n}+\frac{a_{j} a_{j k}}{2}\right] \\
& I_{3}^{\prime}\left(\frac{\left(z-z_{i}\right)^{2}}{r^{2}}\right)=4 \pi A\left(\frac{a_{j}^{2} b_{k}^{2}+a_{k}^{2} b_{j}^{2}+a_{j} a_{k} b_{j} b_{k}}{3 a_{k}^{3}}\right) \sum_{n} \frac{(-1)^{n+1}}{n+3}\left(\frac{a_{j}}{r_{i}}\right)^{n}
\end{aligned}
$$


If $b_{k}=0$,

$$
I_{4}^{\prime}\left(\frac{\left(z-z_{i}\right)^{2}}{r^{2}}\right)=-\frac{4 \pi A b_{j}^{2}}{3 a_{k}} \sum_{n} \frac{(-1)^{n+1}}{n+3}\left(\frac{a_{j k}}{r_{k}}\right)^{n}
$$

If $b_{j}=0$,

$$
\begin{array}{r}
I_{4}^{\prime}\left(\frac{\left(z-z_{i}\right)^{2}}{r^{2}}\right) \\
=\frac{4 \pi A b_{k}^{2}}{3 a_{k}^{3}}\left[3 a_{k} r_{i} \sum_{n . .}(-1)^{n+1}\left(\frac{1}{n+1}-\frac{1}{n+2}\right)\left(\frac{a_{j k}}{r_{k}}\right)^{n}\right. \\
\left.-a_{j}^{2} \sum_{n} \frac{(-1)^{n+1}}{n+3}\left(\frac{a_{j k}}{r_{k}}\right)^{n}-\frac{a_{k} a_{j k}}{2}\right] .
\end{array}
$$

Notice that use of the series forms of two of the integrals, $I_{2}^{\prime}\left[\left(z-z_{i}\right)^{2} / r^{2}\right]$ and $I_{4}^{\prime}\left[\left(z-z_{i}\right)^{2} / r^{2}\right]$, is limited to elements for which either $b_{j}$ or $b_{k}$ equals zero; that is, one side of the element must be oriented in the $r$ direction. If an element has one side oriented at a small nonzero angle with respect to the $z$ direction, no nodal point on the $z$ axis, and no side oriented in the $r$ direction, some other method is required to evaluate these two integrals. In such an event, the two integrals could be evaluated by the application of numerical integration to eqs (13) and (15) for the function $\left(z-z_{i}\right)^{2} / r^{2}$. Computational experience indicates that the series forms of the integrals are efficient for numerical computations within the bounds:

Case 1: $\quad \frac{1}{2}>\frac{a_{k}}{a_{j}}>10^{-6}$ and $\frac{a_{k}}{r_{i}}<10^{-1}$

Case 2: $\quad \frac{1}{2}>\frac{a_{j k}}{a_{j}}>10^{-6}$ and $\frac{a_{j k}}{r_{j}}<10^{-1}$

Case 3: $\quad \frac{1}{2}>\frac{a_{j}}{a_{k}}>10^{-6}$ and $\frac{a_{j}}{r_{i}}<10^{-1}$

Case $4: \quad \frac{1}{2}>\frac{a_{j k}}{a_{k}}>10^{-6}$ and $\frac{a_{j k}}{r_{k}}<10^{-1}$.

For cases falling below the lower bound it is satisfactory to set the integrals equal to zero. For cases falling above either upper bound the general integrals containing the logarithmic terms can be used without difficulty.

The stiffness matrices of the various finite elements. $[k]$ in eqs (8) and (10), are superposed to form the stiffness matrix of the entire structure. This is done by adding the stiffness matrix elements that relate displacements to resulting forces for common or adjacent nodal points of adjacent elements. The resulting stiffness matrix of the entire structure $[K]$ relates the forces applied to the structure $\{F\}$ to the resulting nodal point displacements $\{w\}$ according to the equation

$$
\{\boldsymbol{F}\}=[K]\{\boldsymbol{w}\} .
$$

For problems involving large numbers of unknown displacements, eq (16) can be solved efficiently by iteration. For the work reported here, Gauss-Seidel iteration was used along with overrelaxation and group relaxation as described by Wilson [5]. For the analysis mesh shown in figure 1,250 cycles of iteration resulted in convergence to a state having an absolute sum of unbalanced residual forces of one-half percent of the applied load. This iteration required about $48.8 \mathrm{~s}$. running time on a UNIVAC 1108 computer. Evaluation of the stiffness integrals, eqs (12) through (15), and formation of the stiffness array for the entire structure required only about $6.1 \mathrm{~s}$. computer running time.

\section{Experimental Verification}

To determine how well the finite-element analysis described the structural response of a load cell, a series of laboratory tests were conducted on the loadcell body dimensioned in figure 1 . The load-cell body was made of 18 percent nickel 250 grade maraging steel. For the principal verification tests, an axisymmetric compressive load of $100,000 \mathrm{lbf}(444,800 \mathrm{~N})$ was applied through a small circular area on the top surface and through a circular ring-shaped area on the bottom surface. In a preliminary test, an axisymmetric compressive load of $800,000 \mathrm{lbf}(3,558,400 \mathrm{~N})$ was applied to the load-cell body through two solid cylindrical mild steel blocks. In all tests, the loads were applied by dead weight testing machines.

Strains on the surface of the load-cell body were monitored by fifty-four metal foil strain gages of 0.062 in $(0.16 \mathrm{~cm})$ gage length. Forty-six gages were oriented in the longitudinal direction and located on three lines defined by the intersections of the surface and three radial planes spaced 120 degrees apart. Eight gages were oriented in the circumferential direction and located beside longitudinal gages. Twenty-four longitudinal gages were located in sets of three at common heights and at the three angular locations to detect bending due to load eccentricity.

In the preliminary test, figure 4 , the upper mild steel block was plastically indented by the spherical top surface of the load cell, giving a contact surface of about 1.41 in $(3.58 \mathrm{~cm})$ radius at $800,000 \mathrm{lbf}$ $(3,558,400 \mathrm{~N})$ applied load. The lower mild steel block was used to permit plastic indentation by a small region that protruded about 0.002 in $(0.005 \mathrm{~cm})$ at the center of the bottom surface of the load-cell body. This resulted in continuous contact over the bottom surface upon application of the first 50,000 lbf $(222,400 \mathrm{~N})$ load increment. Recorded longitudinal surface strains due to a load of $800,000 \mathrm{lbf}(3,558,400 \mathrm{~N})$ are plotted in figure 5 . The curves in figure 5 were obtained by finite-element analysis. One curve is for $800,000 \mathrm{lbf}(3,558,400 \mathrm{~N})$ load applied at the centers 


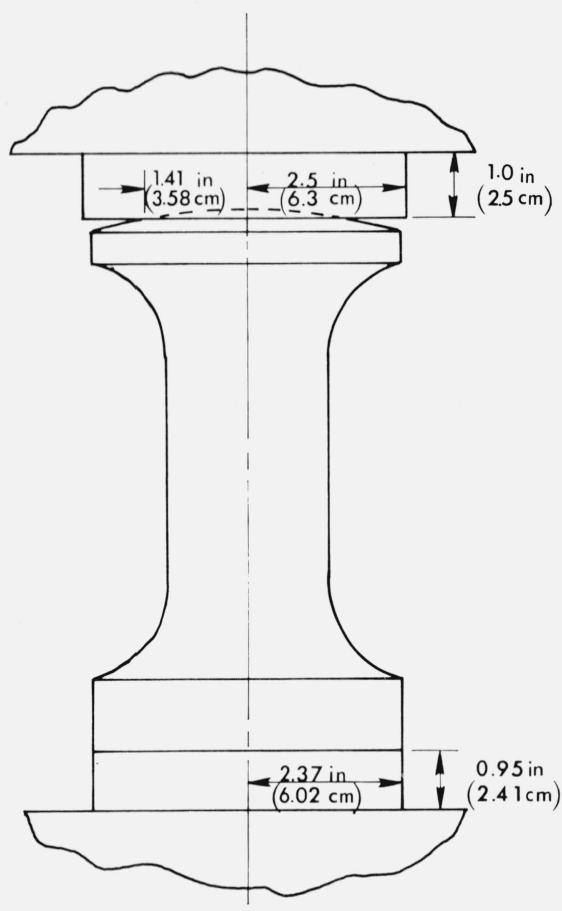

FigURE 4. Setup for applying 800,000 lbf $(3,558,400 \mathrm{~N})$ compressive load in a preliminary test.

of the top and bottom surface. The other curve is for $800,000 \mathrm{lbf}(3,558,400 \mathrm{~N})$ load applied at the radii of the edges of the contact surfaces in the laboratory test, figure 4. Although most of the test data points lie near or are bracketed by the analytical curves, this experiment is clearly insufficient for a precise verification

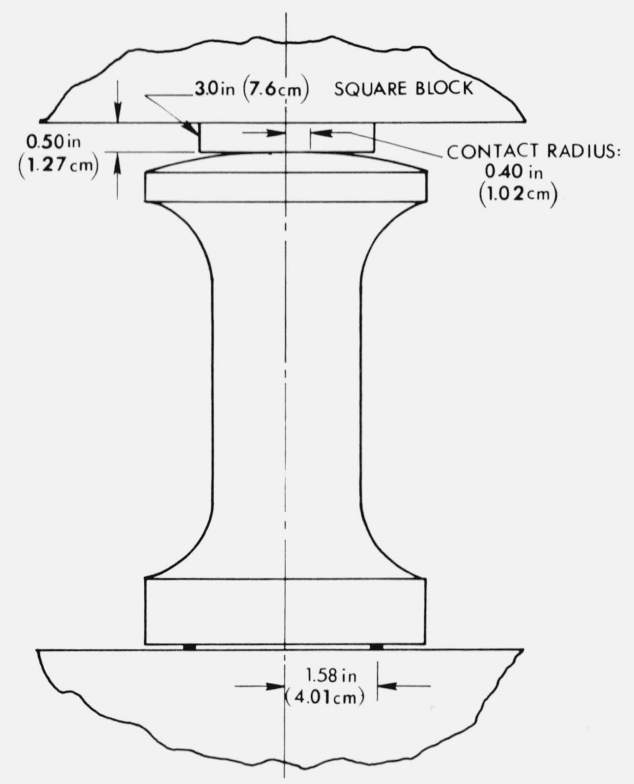

FigURE 6. Setup for applying 100,000 lbf $(444,800 \mathrm{~N})$ compressive load in one of the verification tests.

of the analysis. Figure 5 shows that surface strains can be very sensitive to variations in the distribution of boundary loading.

Figure 6 shows the loading arrangement for one of the principal verification tests. Axisymmetric compressive loads of $100,000 \mathrm{lbf}(444,800 \mathrm{~N})$ were applied to the top surface of the load-cell body through a mild steel block and to the bottom surface through a 0.2 in $(0.5 \mathrm{~cm})$ wide by 0.035 in $(0.088 \mathrm{~cm})$ thick steel ring. Rings with mean radii of $0.40,0.79,1.58$ and 2.27 in $(1.02,2.01,4.01$

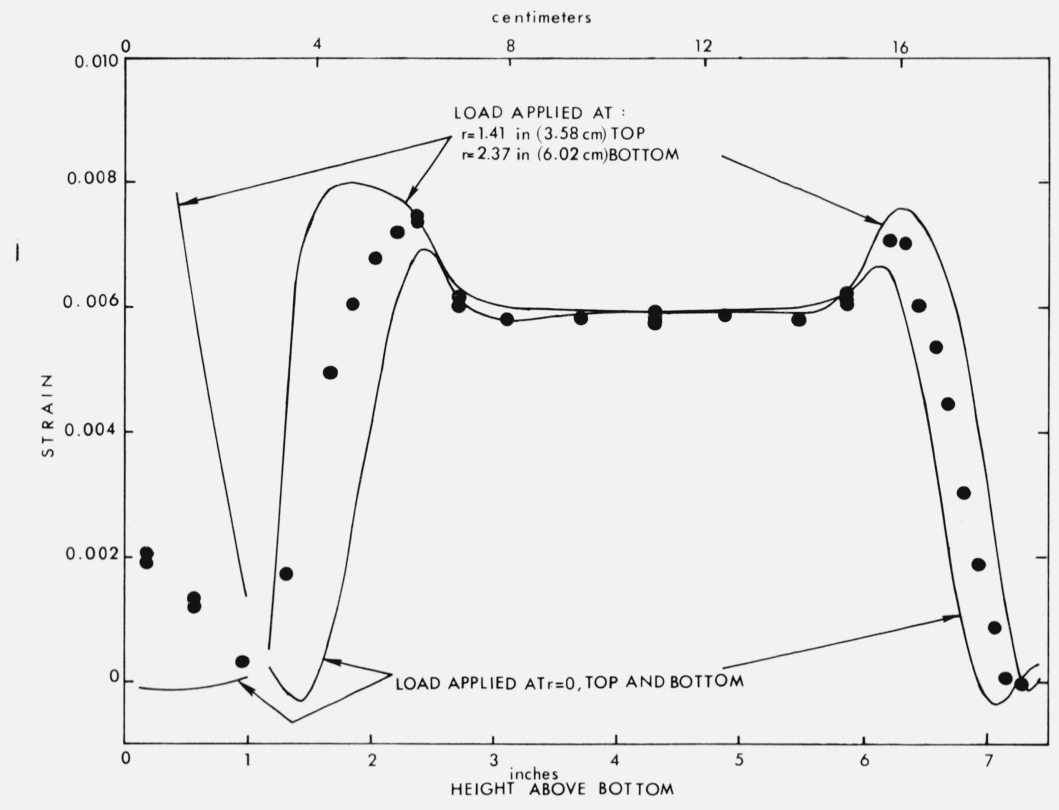

FIGURE 5. Longitudinal surface strains for an applied load of 800,000 lbf $(3,558,400 \mathrm{~N})$ 
and $5.77 \mathrm{~cm}$ ) were used in different tests. Figure 7 shows the recorded longitudinal surface strains for four of these tests (the plotted data points) along with corresponding curves obtained by finite-element analysis. For the finite-element analysis the bottom loading radius corresponds to the mean loading ring radius and the top loading radius corresponds to onehalf the contact surface radius. Much of the scatter in the data is probably due to bending. The value of the modulus of elasticity used in the finite-element analysis was $26.8 \times 10^{6} \mathrm{lb} / \mathrm{in}^{2}\left(1.85 \times 10^{10} \mathrm{~N} / \mathrm{m}^{2}\right)$. This value was computed by averaging, for several tests, the data obtained from the three strain gages located at $z=4.31$ in $(10.95 \mathrm{~cm})$. This particular data was very repeatable and bending errors were essentially cancelled by the averaging. Admittedly, this procedure for determining the elastic modulus imposes good agreement between analysis and experiment in the region near $z=4.31$ in $(10.95 \mathrm{~cm})$. A Poisson's ratio of 0.3 , a value listed in the literature of steel producers for 18 percent nickel 250 grade maraging steel, was used in the finite-element analysis. The generally good agreement between the analysis and this test data for the entire length of the load-cell body (fig. 7) indicates that the analysis would be suitable for the study of such subjects as stress concentration and the optimum geometry of material test specimens and fixtures.

For use as a force transducer, a load-cell body of the type tested is ordinarily instrumented with strain gages over only a small region near mid-height. An important consideration in the design of such a load cell is the change in surface strain sensed by these strain gages due to a change in the distribution of boundary loading. A portion of the curves and test data in figure 7 that bear directly on this question are replotted to a much greater scale in figure 8 . The test data points in figure 8 have been averaged from the strain readings of sets of three gages at different angular locations to minimize bending errors. Figure 9 is a plot of the differences between surface strain for a particular radius of load at the bottom surface and the corresponding strain for a 2.27 in $(5.77 \mathrm{~cm})$ radius of load. Figures 8 and 9 indicate very good agreement between the finite-element analysis and the test results at the three strain gage locations represented. The greatest difference between the analytical and averaged experimental values represented in figure 9 is $2 \times 10^{-6}$. This difference approache the resolution of the digital indicator $\left(1 \times 10^{-6}\right)$ used in the laboratory tests. This data verifies the accuracy of the analysis in predicting the sensitivity of such a load cell to changes in the distribution of boundary loading.

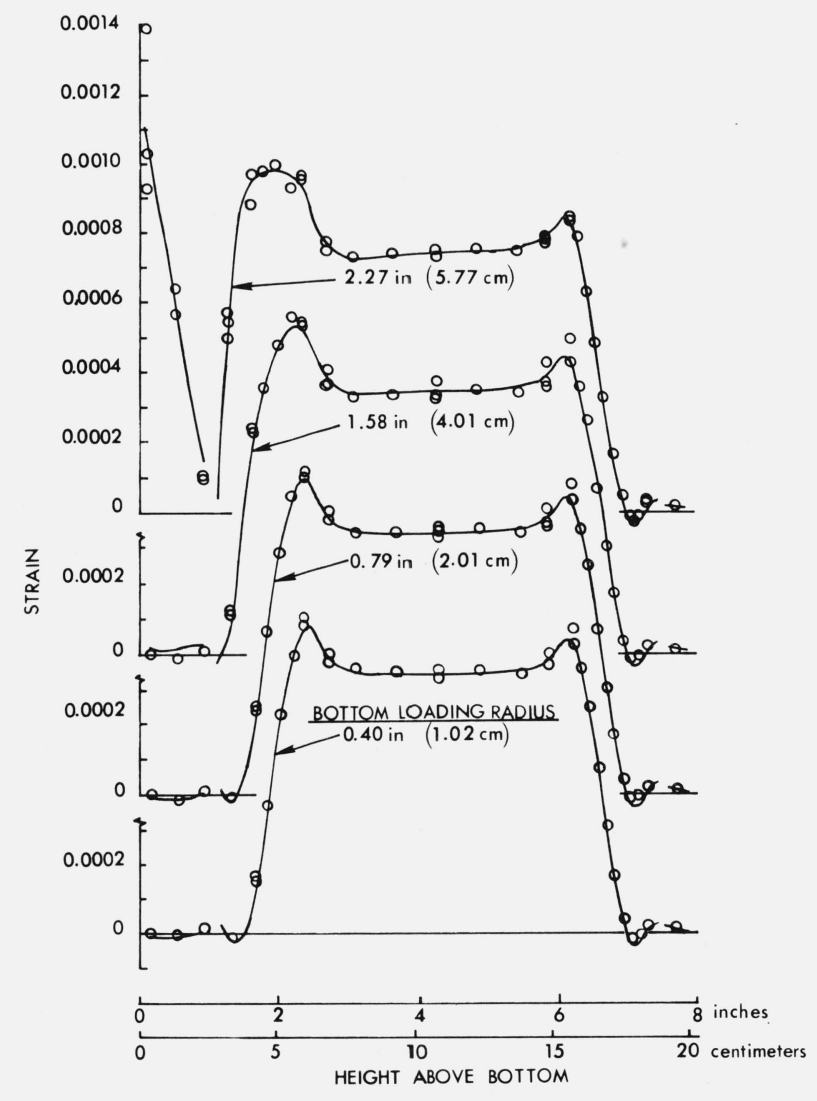

FIGURE 7. Longitudinal surface strains for an applied load of 100,000 lbf $(444.800 \mathrm{~N})$.

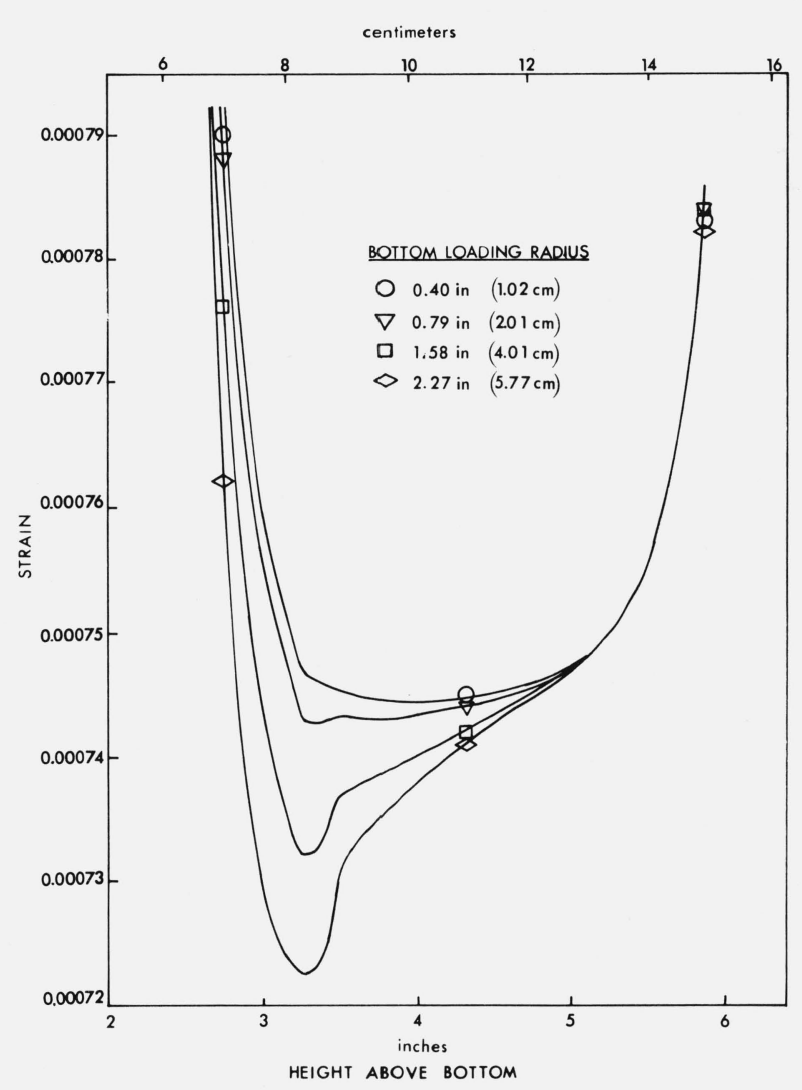

FIGURE 8. Longitudinal surface strains averaged for sets of three gages at common heights and different angular locations. 


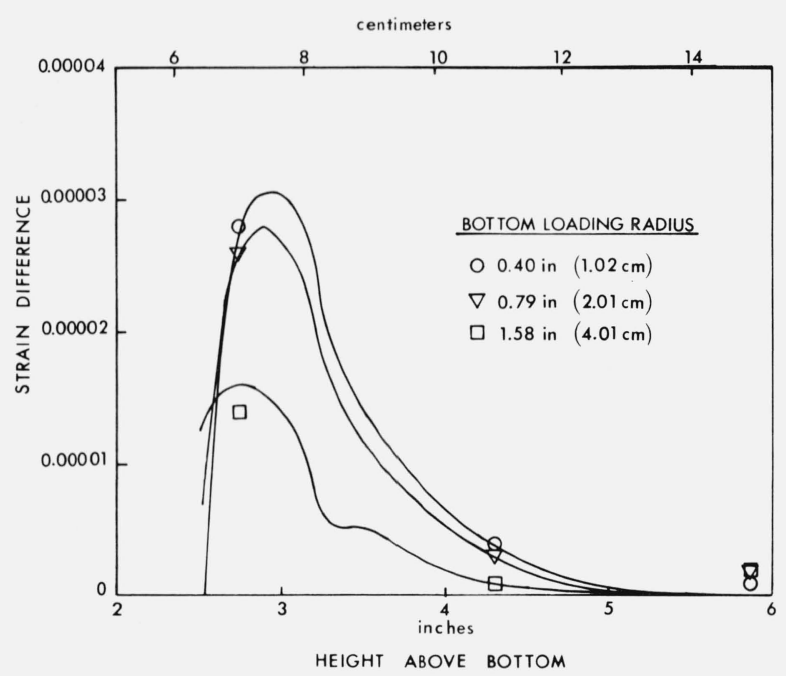

FIGURE 9. Difference between surface strain for a particular loading radius and the corresponding strain for a loading radius of 2.27 in $(5.77 \mathrm{~cm})$.

\section{Conclusion}

A finite-element structural analysis has been formulated for application to problems in which geometry and loading are axisymmetric and materials are isotropic elastic. An attempt to minimize restrictions imposed on the shape and orientation of the triangular elements employed has been largely successful. This facilitates use of the analysis, with automatic finite- element mesh generation, in parameter or optimization studies.

An experimental verification of the analysis has been obtained by a series of tests conducted on a loadcell body. In these tests the magnitude and distribution of boundary loading was known within narrow limits. Strains measured on the surface of the load-cell body were in good agreement with the finite-element analysis.

C. H. Melton was largely responsible for instrumenting the load cell for the measurement of surface strains.

\section{References}

[1] Clough, R. W., The finite element method in structural mechanics, chapter 7 of Stress Analysis, Eds. Zienkiewicz, O. C. and Holister, G. S. (Wiley, 1965).

[2] Clough, R. W., and Rashid, Y., Finite element analysis of axisymmetric solids, J. Engr. Mech. Div. ASCE 91, No. EM 1 (Feb. 1965)

[3] Rashid, Y. R., Solution of elasto-static boundary value problems by the finite element method, Ph. D. Dissertation, U. of Cal. Berkely (1964).

[4] Rashid, Y. R., Finite element analysis of axisymmetric composite structures, AEC Res. and Dev. Report GA-6303 (June 4, 1965).

[5] Wilson, E. L., Finite element analysis of two-dimensional structures, Structures and Materials Research Report No.63-2, U. of Cal. Berkeley (June 1963).

[6] Timoshenko, S. and Goodier, J. N., Theory of Elasticity (McGrawHill, 1951).

[7] Zienkiewicz, O. C., The Finite Element Method in Structural and Continuum Mechanics (McGraw-Hill, 1967).

[8] Peirce, B. O., A Short Table of Integrals (Ginn, 1929).

(Paper 75C3\&4-322) 